\title{
Reemplazo parcial o total del arco aórtico. Experiencia en 23 pacientes
}

\author{
Manuel J Irarrázaval LI, Sergio Morán V, Ricardo Zalaquett S, \\ Pedro Becker R, Cristian Baeza $\mathbf{P}^{1}$, Jorge Urzúa U², \\ Guillermo Lema $F^{3}$, Roberto Canessa B, Gastón Chamorro S, \\ Sandra Braun J, Samuel Córdova A, Bernardita Garayar P.
}

\author{
Partial or total replacement \\ of the aortic arch. \\ Experience in 23 patients
}

Background: Surgery of the aortic arch is a very complex procedure since it requires protective strategies for the brain, heart and rest of the body. Aim: To communicate our experience in the first 23 total or partial replacements of aortic arch. Material and methods: Retrospective search in the database of the Cardiovascular Surgery Unit for patients subjected to partial or total replacement of the aortic arch since 1998. Results: Between 1988 and 2002, 23 patients were operated. Seventeen had aortic dissection (10 acute and 7 chronic), five had an atherosclerotic aneurysm and one had a traumatic lesion. Thirteen patients were subjected to a replacement of the arch plus ascending aorta, six to a replacement of the arch plus descending aorta and four to a replacement of the arch, ascending and descending aorta. Seven patients had previous operation of the thoracic aorta. Arterial perfusion was done via the femoral artery, axillary artery or a combination of both. A hypothermic circulatory arrest was induced in 22; it was associated with cerebral retro perfusion alone in 8 patients, antegrade cerebral perfusion in 5; isolated or associated axillary perfusion was used in five patients. In seven, procedures on the aortic or mitral valve, or coronary artery operations were added. Operative mortality was $26 \%, 3$ of the 8 patients operated as an emergency and 3 of 15 elective operations. There was no mortality among those without dissection and of 7 chronic dissections, one died. All patients were followed for an average of 45 months. Two patients required reinterventions on the aorta and one for colon cancer. There was one late death of unknown cause. Postoperative complications were agitation, bleeding and temporary vocal cord dysfunction. Conclusions: There is a learning curve, where more extensive operations, particularly those done as emergency or for dissections, had an increased operative risk (Rev Méd Chile 2006; 134: 575-80). (Key words: Aorta, thoracic; Aortic aneurysm, thoracic; Hipothermia; Perfusion)

\footnotetext{
Recibido el 12 de julio, 2005. Aceptado el 8 de noviembre, 2005.

Departamentos de Enfermedades Cardiovasculares y Anestesia Cardiovascular, Facultad de Medicina, Pontificia Universidad Católica de Chile.

${ }^{1}$ Actualmente en Clínica Alemana

${ }^{2}$ Actualmente en Universidad Santo Tomás

${ }^{3}$ Departamento Anestesia Cardiovascular, PUC de Chile
}

Correspondencia a: Manuel José Irarrázaval Ll. Marcoleta 367, 6 piso. Teléfonos 633 3030-354 3231. Fax 6390108.

E-mail: manuelj@med.puc.cl 
$\mathrm{E}^{\mathrm{n}}$ 1957, De Bakey y cols comunicaron el primer reemplazo exitoso de un aneurisma del arco aórtico con un homoinjerto ${ }^{1}$. El mismo año, Niazi y Lewis ${ }^{2}$ introdujeron la hipotermia profunda y el paro circulatorio. Sin embargo, esta técnica no fue popularizada para su uso en pacientes adultos con aneurismas del arco aórtico hasta 1975, cuando Griepp y cols publicaron su experiencia ${ }^{3-5}$.

El arco aórtico se define como el segmento de la aorta torácica, comprendido entre el origen proximal del tronco innominado y el origen distal de la arteria subclavia izquierda ${ }^{3}$. Este segmento aórtico es un lugar estratégico fundamental, por cuanto vincula la circulación sistémica con la circulación cerebral y de las extremidades superiores, a través de los llamados troncos supraaórticos (tronco innominado, carótida izquierda y subclavia izquierda). La ubicación anatómica de este segmento aórtico le confiere un especial desafío al acceso quirúrgico, ya que está rodeado de estructuras vitales, como la tráquea, otros vasos sanguíneos intratorácicos, el esófago y estructuras nerviosas como el recurrente laríngeo y los nervios frénicos. El acceso quirúrgico a este segmento obliga a la interrupción momentánea de toda la circulación sistémica, incluida la irrigación a órganos nobles con baja tolerancia a la isquemia, como el sistema nervioso central.

Se estima que aproximadamente $1 / 3$ de los aneurismas de la aorta afectan exclusivamente a la aorta torácica y otro tercio afectan el segmento tóraco-abdominal ${ }^{6}$. No hay datos claros sobre la incidencia de aneurismas aislados del arco aórtico, puesto que desde el punto de vista patológico, ninguna de las enfermedades que afectan a este segmento de la aorta, ya sea aneurisma puro o disecante, se limita exclusivamente al arco, siendo habitualmente éste parte de un compromiso más extenso que involucra la aorta ascendente 0 descendente y en ocasiones a los tres sectores simultáneamente.

Comunicamos la experiencia en el Hospital Clínico de la Universidad Católica con las primeras 23 operaciones de reemplazo parcial o total del arco aórtico, mediante el uso de circulación extracorpórea, paro circulatorio en hipotermia profunda (PCHP) y algunas técnicas específicas de protección cerebral.

\section{PACIENTE Y MÉTODO}

Este es un estudio observacional descriptivo no comparado, de una serie clínica sometida a cirugía de reemplazo parcial o total del arco aórtico.

En la Base de Datos del Servicio de Cirugía Cardiovascular, se identificaron todos los pacientes sometidos a cirugía en la aorta torácica desde 1988 hasta diciembre 2004. Del subgrupo de 292 pacientes sometidos a cirugía de la aorta torácica, 26 casos cumplen con la definición de reemplazo parcial o total del arco aórtico.

En este estudio consideramos como cirugía del arco aórtico aquella en la cual se reemplazó el arco aórtico en forma parcial o total, siendo necesaria una planificación especial respecto del uso de paro circulatorio hipotérmico, para la protección del cerebro, del corazón y del resto del organismo durante dicho período.

Se excluyeron 3 pacientes sometidos a reemplazo del arco aórtico en el año 2004, con el propósito de tener una serie con al menos un año de seguimiento. Se conformó así un grupo de 23 pacientes para el estudio. Los datos clínicos se obtuvieron de las fichas clínicas, y el seguimiento se completó en todos, mediante control médico habitual o contacto telefónico, para aquellos con más de un año desde el último control.

Hubo 17 pacientes hombres $(73,9 \%)$ y 6 mujeres (26,1\%). La edad promedio del grupo fue $58,3(58,3 \pm 15,7)$ años, con un rango que va entre 26 y 79 años. Si bien no se hicieron estudios genéticos, se registraron 3 (13\%) pacientes con características clínicas e historias familiares compatibles con enfermedad de Marfan.

Características clínicas. En relación con el diagnóstico patológico, 1 paciente $(4,3 \%)$ fue tratado por una lesión traumática del arco aórtico, 5 pacientes $(21,7 \%)$ tenían un aneurisma fusiforme ateroesclerótico y 17 (73,9\%) tenían un aneurisma disecante. Todos los diagnósticos se hicieron por ecocardiografía, scanner con contraste o resonancia nuclear magnética.

De los 23 pacientes, 11 (48\%) fueron operados con criterio de urgencia. De éstos últimos, 10 correspondieron a disecciones agudas ( $<15$ días) y 1 correspondió a una lesión traumática del arco. Todos ellos se operaron dentro de las primeras 48 $\mathrm{h}$ de diagnosticado el cuadro. En 7 (30\%) de los 23 
pacientes, estaba el antecedente de una cirugía cardíaca previa: uno tenía una esternotomía exploradora dos días antes, otro había sido sometido ya a un reemplazo de la aorta tóraco-abdominal por una disección crónica y otro a un reemplazo de la aorta ascendente. En los otros 4 pacientes se habían hecho reemplazos compuestos de aorta ascendente y prótesis aórtica, entre 4 meses y 5 años antes de la cirugía de reemplazo del arco aórtico. Adicionalmente, 1 paciente fue sometido a una angioplastia coronaria percutánea los días previos.

La vía de abordaje preferida, en la gran mayoría, fue la esternotomía media tradicional, utilizada en 17 (74\%) pacientes. La toracotomía izquierda se utilizó en 4 pacientes y en 2 pacientes se utilizó una esternotomía en $\mathrm{T}$ hacia el segundo espacio intercostal izquierdo.

En los pacientes que requirieron reemplazo completo del arco aórtico, los troncos supraaórticos se manejaron como un botón, suturada a la cara lateral y cefálica de la prótesis de dacron. Esta anastomosis se realizó después de la anastomosis al segmento proximal de la aorta descendente. En los casos en que había compromiso aneurismático de los troncos supraaórticos, éstos se trataron individualmente con prótesis de dacron separadas, según fue necesario. Aquellos pacientes cuya enfermedad principal estaba en la aorta ascendente y se extendía por contigüidad hacia el arco, requiriendo un reemplazo parcial o hemiarco (situación frecuente en los casos de disección aguda de la aorta ascendente), se trataron mediante transección del arco al nivel de la base del

\section{Tabla 1. Procedimientos quirúrgicos}

\begin{tabular}{|lrr|}
\hline Extensión del reemplazo & $\mathrm{N}$ & $\%$ \\
\hline Ao ascendente + arco & 13 & 50,5 \\
Arco + Ao descendente & 6 & 26,1 \\
Ao ascendente + arco + Ao descendente & 4 & 17,4 \\
\hline Procedimientos concomitantes & & \\
\hline Cirugía de revasc. miocárdica & 4 & 18 \\
Reemplazo valvular Ao & 1 & 4 \\
Reparación valvular Ao & 1 & 4 \\
Anuloplastia mitral & 1 & 4 \\
Total de procedimientos asociados & 7 & 30 \\
\hline
\end{tabular}

tronco innominado, por la curvatura mayor, y proximal a la aorta descendente, por la curvatura menor. De esta manera se obtenía una boca anastomótica biselada, con una anastomosis única. La extensión del reemplazo del segmento aórtico se determinó en cada caso durante el acto quirúrgico, en correlación con las imágenes disponibles en el preoperatorio (TAC, RNM, ecocardiograma) y en los hallazgos operatorios de la extensión de la ruptura intimal. Así, además del reemplazo parcial o total del arco, en cada uno de los pacientes, se realizaron reemplazos de los segmentos aórticos inmediatamente adyacentes según se describe en la Tabla 1. Siete pacientes (30\%) tuvieron, además, otros procedimientos asociados como se describen también en la Tabla 1.

En 19 pacientes se utilizaron prótesis precoaguladas rectas simples de Hemashield ${ }^{\circledR}$ para el reemplazo, en 3 pacientes se implantaron tubos valvulados compuestos y en 1 paciente, con una úlcera penetrante, se utilizó un parche del mismo material.

El acceso arterial para la circulación extracorpórea se determinó según la preferencia del cirujano y las condiciones particulares de cada paciente. La arteria femoral se utilizó en 12 (52\%) pacientes, la arteria axilar derecha exclusiva se utilizó en 5 (22\%) pacientes (a través de una prótesis de Hemashield ${ }^{\circledR}$ de $8 \mathrm{~mm}$ suturada a la cara anterior de la arteria axilar) y en otros 5 (22\%) pacientes se utilizó una combinación de canulación femoral y aorta ascendente, ápex del ventrículo izquierdo o arteria axilar. En un paciente (2\%) se canuló una prótesis axilofemoral previa.

En cuanto a las técnicas de perfusión para protección cerebral, en 4 (17,3\%) pacientes se usó paro circulatorio en hipotermia profunda (PCHP) exclusivamente, en 8 (34,7\%) pacientes, el PCHP se asoció a perfusión cerebral retrógrada (PCR) aislada y en $2(8,6 \%)$ pacientes se utilizó perfusión anterógrada axilar exclusiva. En $4(17,3 \%)$ pacientes se hizo una combinación de PCR y perfusión anterógrada axilar. Finalmente, en $4(17,3 \%)$ pacientes se hizo combinación de PCR y perfusión cerebral anterógrada directa en los ostia supra aórticos.

El protocolo utilizado para la realización del PCHP se describe en la Tabla 2. En cuanto al circuito de perfusión, se estableció con bomba de rodillo y un oxigenador de membrana en línea (Terumo sx-18 ${ }^{\circledR}$ ). El cebado del circuito se realizó con solución cristaloide $2 / 3$ de ringer lactato y 1/3 
Tabla 2. Protocolo utilizado en PCH P y protección cerebral

\begin{tabular}{|ll|}
\hline Todos los pacientes & Temperatura menor a $20^{\circ} \mathrm{C}$ \\
& Cabeza envuelta en hielo \\
& Cebado de la bomba con Mannitol antes del paro \\
& Control de PH con Alfa-stat \\
& Campo quirúrgico saturado con irrigación de CO2 \\
& Tiopental $10 \mathrm{mg} / \mathrm{kg} 5 \mathrm{minutos}$ antes del paro \\
& Metilprednisolona $30 \mathrm{mg} / \mathrm{kg} 30$ a $60 \mathrm{~min}$ antes del paro \\
& Aprotinina: \\
& -2 millones de U antes de iniciar la CEC \\
& -2 millones de U en el cebado del circuito \\
& -500.000 U c/hora hasta el final de la cirugía \\
& Uso de Cell-saver ocasional \\
& Canulación subclavia a través de un injerto lateral de Hemashield ${ }^{\circledR}$ \\
& de 8 mm \\
& Oclusión tronco innominado y/o carotídea con catéter de plegia \\
Perfusión arterial anterógradada & retrógrada \\
& Presión mantenida entre 40 y 60 mm de Hg \\
A través de la vena cava superior cinchada bajo la vena azygos \\
Perfusión cerebral retrógrada
\end{tabular}

de manitol. El volumen total se determinó según superficie corporal y hematocrito del paciente, entre 600 cc y 1.500 cc (aplicando técnicas de autocebado, cebado retrógrado o drenaje venoso asistido por vacío). Se utilizó un set de líneas estándar: línea venosa de $1 / 2$ pulgada y arterial de $3 / 8$ pulgada. A esta última se agregó un conectador en \&y para una segunda línea arterial a ser usada en caso de perfusión cerebral retrógrada (a través de la cava superior), anterógrada (a través de la arteria axilar o directamente en los ostia de los vasos supraaórticos) o ambos. El paro circulatorio hipotérmico se alcanzó mediante un enfriamiento lento a razón de 1 grado por minuto hasta alcanzar $18^{\circ} \mathrm{C}$, registrados por sondas nasofaríngea y rectal. La técnica completa de perfusión, paro circulatorio y perfusión cerebral retrógrada, además de los diagramas de perfusión, se describen en una publicación previa de este mismo grupo ${ }^{7}$.

En relación con el tiempo de PCHP, en 3 pacientes operados antes de 1995 no se encontró registro de la duración del PCHP. En dos pacientes el PCHP fue de 120 min y ambos fallecieron durante la operación por problemas técnicos y anatómicos que no pudieron ser corregidos adecuadamente. En los otros 18 pacientes, el tiempo promedio de PCHP fue de $39 \pm 20,06$ (15 SD) min, con un intervalo entre 14 y $87 \mathrm{~min}$. Hubo 2 pacientes en que el PCHP se extendió por sobre una hora (85 y $87 \mathrm{~min}$ ), en ambos casos se realizó perfusión anterógrada intermitente vía arteria axilar.

Para la evaluación postoperatoria del compromiso neurológico, se definió accidente vascular encefálico (AVE) postoperatorio como el déficit neurológico focal o difuso que no estaba presente en el preoperatorio. Electroencefalograma y evaluación neurológica se obtuvo precozmente en todos los pacientes que cumplieron con estos criterios, y en aquellos pacientes hemodinámicamente estables, se les realizó un TAC o una RNM de cerebro durante la hospitalización.

\section{RESULTADOS}

La mortalidad hospitalaria (hasta 30 días o el alta) de toda la serie fue $26 \%$ ( 6 pacientes). Tres de estos pacientes fallecieron en pabellón y de los 3 restantes, uno falleció por falla multiongánica a los 15 días de la operación y los otros dos pacientes fallecieron precozmente en el postoperatorio debido a gran inestabilidad hemodinámica. Estos dos pacientes fallecieron en el postoperatorio inmediato por lo que no se logró una evaluación 
neurológica que permitiera confirmar ni descartar lesión neurológica.

De los 11 pacientes operados en condiciones de urgencia, fallecieron 3 (27\%), de los 12 pacientes operados en condiciones electivas fallecieron 3 (25\%). Sin embargo, en el subgrupo de 11 pacientes operados en forma electiva sin disección aguda, falleció sólo uno.

Desde el punto de vista de la patología, de los 5 pacientes con aneurisma no disecante, no falleció ninguno. De los 17 pacientes con disección fallecieron 5; 4 de los 10 pacientes operados por disección aguda y 1 de los 7 pacientes operados por disección crónica.

Dentro de las complicaciones postoperatorias, no se registró AVE en los sobrevivientes y hubo 5 pacientes con agitación psicomotora sin secuelas, 1 de ellos debió ser conectado a ventilación mecánica por $48 \mathrm{~h}$. No se logró establecer una relación entre tiempo de PCHP o tiempo quirúrgico total con relación a este subgrupo de pacientes. Dos pacientes tuvieron sangrado postoperatorio que debió ser reexplorado, y finalmente, 3 pacientes presentaron disfonía postoperatoria. En dos de ellos hubo una resolución completa y el paciente restante persistió con voz bitonal.

El seguimiento alejado se completó en los 17 (100\%) sobrevivientes por un período que va entre 11 y 139 meses, con un promedio de $45 \pm$ 43,5 meses. Sólo se registró la muerte alejada de un paciente, cuya causa se desconoce.

En cuanto a la morbilidad tardía, 1 paciente requirió una hemicolectomía por cáncer 2 años después de la operación aórtica, estando en buenas condiciones en la actualidad. Un paciente requirió 1 año después de la operación del arco, un reemplazo de la aorta ascendente por dilatación progresiva de ese segmento, y a otro paciente, se le instaló una endoprótesis distal a la subclavia izquierda por una redisección de aorta descendente, con buen resultado alejado.

\section{DisCUSIÓN}

La cirugía de la aorta ha sido desde sus inicios un gran desafío para los cirujanos cardiovasculares, por su complejidad y elevada morbimortalidad. Durante la última década se han hecho importantes avances, tanto de las técnicas quirúrgicas como de perfusión, lo que ha significado una disminución significativa del riesgo quirúrgico. Sin embargo, con la aparición de casos de creciente complejidad, se hace necesario seguir investigando nuevas técnicas de preservación cerebral, que permitan mayores tiempos de isquemia para realizar reparaciones más complejas sin aumentar el riesgo de secuelas neurológicas.

$\mathrm{Si}$ consideramos el total de cirugías realizadas sobre la aorta torácica hasta diciembre de 2003 (cierre del estudio), éstas fueron 272, de las cuales 23 corresponden a procedimientos de reemplazo parcial o total del arco aórtico, algo menos de $10 \%$, lo que es similar a lo comunicado en otras series $^{8}$. De los 6 pacientes que fallecieron, en todos se pudo identificar con claridad razones técnicas derivadas de la complejidad de las disecciones, que se asociaron a procedimientos muy prolongados que tienen como consecuencia sangrado precoz o disfunción ventricular que llevan a su fallecimiento pocas horas después de la operación. Sólo uno de estos pacientes tuvo un postoperatorio más prolongado con falla multiorgánica asociado a insuficiencia respiratoria.

La cirugía que comprende el arco aórtico es técnicamente mucho más compleja y heterogénea, requiriendo, por lo tanto, la capacidad de abordajes por distintas vías, que van desde la esternotomía clásica, la estemotomía en $\mathrm{T}$ a la bitoracotomía anterior. Respecto a las técnicas de perfusión arterial, el cirujano debe estar preparado para ofrecer distintas estrategias, de acuerdo a la situación anatómica del paciente, considerando la perfusión femoral, axilar, prótesis antigua, ápex del ventrículo izquierdo. La protección cerebral también tiene distintas estrategias, destacando la retroperfusión cerebral a través de la vena cava superior, la perfusión axilar con oclusión temporal de la raíz del tronco innominado y la protección cerebral con perfusión cerebral directa a través de cánulas en los orificios innominados y carotídeos.

Este es un grupo de pacientes de condiciones clínicas muy graves, frecuentemente requiriendo reoperaciones por procesos disecantes previos.

En nuestra limitada experiencia local, en las operaciones más extensas, esto es, que requirieron simultáneamente reemplazo de la aorta ascendente, arco y descendente, los riesgos perioperatorios son significativos. Cuando la operación fue más limitada, a la aorta ascendente y arco o descenden- 
te y arco, los resultados fueron más satisfactorios; hay una tendencia a menor riesgo en las operaciones electivas que en las de urgencia y en las no disecantes que en las disecantes.

Uno de los aspectos más importantes a discutir en relación con esta serie, es la técnica de preservación utilizada y los resultados obtenidos. El desafío de proteger adecuadamente el cerebro durante este tipo de cirugías, ha llevado a diversos grupos a desarrollar protocolos multimodales para la realización del $\mathrm{PCHP}^{8-11}$. En esta serie de pacientes, al igual que en la publicación anterior ${ }^{7}$, no encontramos incidencia de AVE entre los sobrevivientes, habiendo registrado sólo algunos casos de despertar tardío en relación con las cirugías más prolongadas y pacientes de mayor riesgo. El mejor dominio de las técnicas quirúrgicas y de perfusión logró que en aquellos pacientes con lesiones de mayor complejidad, mediante el uso combinado de técnicas de perfusión retrógrada y anterógrada se prolongaran, los tiempos de paro circulatorio sin

\section{REFERENCIAS}

1. De Bakey ME, Crawford ES, Cooley DA, Morris GC JR. Successful resection of fusiform aneurysm of aortic arch with replacement by homograft. Surg Gynecol Obstet 1957; 105: 657-64.

2. NiAZI SA, LEWIS FJ. Profound hypothermia in man; report of a case. Ann Surg 1958; 147: 264-6.

3. Kouchoukos NT, DougenIs D. Surgery of the thoracic aorta. N Engl J Med 1997; 336: 1876-88.

4. Gross RE, BшL AHJ, PeiRCE ECI. Methods for preservations and transplantation of artrial grafts: observations on arterial grafts in dogs: reprt of transplantation of preserved arterial grafts in 9 human cases. Surg Gynecol Obstet 1949; 88: 689-701.

5. Cosew JS, Buket S, DJukanovic B. Aortic arch operation: current treatment and results. Ann Thorac Surg 1995; 59: 19-26; discussion 26-7.

6. Perko MJ, Norgaard M, Herzog TM, Skov Olsen P, Shroeder TV, Petterson G. Unoperated aortic aneurysm: a survey of 170 patients. Ann Thorac Surg 1995; 59: 1204-9.

7. Zalaquett R, Irarrázaval MJ, Morán S, Muñoz C, GaRAYAR B, Becker P et al. [Retrograde cerebral perfusion during circulatory arrest with deep hypothermia. A new technique for brain protec- necesariamente aumentar la morbilidad neurológica. En el año 1994, Kouchoukos y cols ${ }^{12}$ publicaron el uso de esta técnica combinada de perfusión, que nosotros empleamos satisfactoriamente en $8(35 \%)$ de nuestros pacientes.

Esta es una serie pequeña y que representa la curva de aprendizaje de la técnica tanto quirúrgica, como de perfusión y protección cerebral. Las principales complicaciones fueron agitación psicomotora, probablemente vinculada al período de isquemia cerebral, que no ha dejado secuelas neurológicas detectables al examen neurológico; el sangrado, que requirió reexploración en 3 pacientes y la disfonía, probablemente vinculada a lesión temporal del recurrente laríngeo, que en 2 de ellos fue reversible a los 6 meses.

El seguimiento alejado de estos pacientes, si bien muestra, al igual que en otras series, la necesidad de procedimientos en otros territorios, ofrece una excelente expectativa de sobrevida en el contexto de una patología inicial de gravedad.

tion in surgery of ascending aorta and aortic arch]. Rev Méd Chile 1995; 123: 1489-98.

8. Svensson LG, Nadolny EM, Kimmel WA. Multimodal protocol influence on stroke and neurocognitive deficit prevention after ascending/arch aortic operations. Ann Thorac Surg 2002; 74: 2040-6.

9. Crawford ES, Svensson LG, Cosem JS, Safi HJ, Hess KR. Surgical treatment of aneurysm and/or dissection of the ascending aorta, transverse aortic arch, and ascending aorta and transverse aortic arch. Factors influencing survival in 717 patients. J Thorac Cardiovasc Surg 1989; 98: 659-73; discussion 673-4.

10. Davis EA, Gilunov AM, Cameron DE, Reitz BA. Hypothermic circulatory arrest as a surgical adjunct: a 5-year experience with 60 adult patients. Ann Thorac Surg 1992; 53: 402-6; discussion 406-7.

11. Safi HJ, Milier ChC, Estera AL, Huynh TT, RubensTEIN FS, SubRamaniam MH ET AL. Staged repair of extensive aortic aneurysms: morbidity and mortality in the elephant trunk technique. Circulation 2001; 104: 2938-42.

12. Kочсноuкоs NT. Adjuncts to reduce the incidence of embolic brain injury during operations on the aortic arch. Ann Thorac Surg 1994; 57: 243-5. 